\title{
PlayChemy: Educational Application in Augmented Reality for Equating Redox Equations
}

\author{
Maria Nistor-Brana \\ Technical University of Cluj- \\ Napoca \\ Str. G. Barițiu 28, 400027, \\ Cluj-Napoca, Romania \\ nistorbranaleyahoo.com
}

\author{
Adrian Sabou \\ Technical University of Cluj- \\ Napoca \\ Str. G. Barițiu 28, 400027 , \\ Cluj-Napoca, Romania \\ adrian.saboules.utcluj.ro
}

\begin{abstract}
Augmented reality combines the virtual world with the real world by superimposing various digital objects on top of real objects. This type of technology brings many benefits to education, including making it easier to accumulate new information or boosting memory capacity. We propose to develop an augmented reality-based game with the aim of facilitating learning and understanding of methods for determining oxidation numbers and equating redox equations. The paper presents the development steps that have been followed in the realization of this project, from the definition of the application context to the actual implementation.
\end{abstract}

\section{Author Keywords}

Augmented reality; Educational application; Chemistry; Unity

\section{ACM Classification Keywords}

H.5.2. User Interfaces.

DOI: 10.37789/ROCHI.2021.1.1.10

\section{INTRODUCTION}

Recently, video games have become increasingly popular, especially in the educational field. Although the main purpose of the game is entertainment, it facilitates the development of the cognitive system, memory skills and creativity. For example, strategy games help to stimulate motivation, puzzle games help to develop logical thinking and reasoning, sports or dynamic games help to relieve stress and facilitate psychomotor coordination. [1].

Among the most commonly used devices for gaming are computers, laptops, tablets, phones and consoles. Statistics show that globally the most used device for video games is the phone, followed by the computer and then the console. [2]. Mobile technology has developed very quickly, so most of the functions on the computer have been transferred to mobile devices. Because of their ease of use, smartphones are some of the most widely used mobile devices today.

One type of app that allows the real world to be connected to the virtual world is the AR (Augmented Reality) app. Games using AR technology are much more attractive than normal games, they involve an interactive experience with a realworld environment. An AR application is defined as a system that has the following three characteristics: it combines the virtual world with the real world, allows real-time interaction and allows $3 \mathrm{D}$ objects to be registered in the real and virtual world [3]. The principle of this technology is based on the following steps: capturing the image of the real world, recognising objects and superimposing objects on the real world. In the image capture step, the scene where the virtual objects will be processed is set up.

Equating a redox equation is much more complex than equating a standard chemical equation. One method of equating equations of this type is the oxidation number method. These numbers play an important role in the oxidation-reduction process, and their determination is based on a well-defined set of rules. Linear algebra can be used to determine the final stoichiometric coefficients of a redox equation. This avoids increasing the processing time for those equations that are more complex.

The way AR technology creates a virtual environment, full of colour, sound and fascinating objects, where the user has complete control over them, can be used as a stimulus in the learning process for both children and adults. It brings many benefits in the field of education, including ease of learning new information, motivation, development of memory capacity and imagination. AR applications are very versatile and can be used in different fields: biology, physics, or geography.

The main objective of this project is to use augmented reality to implement an educational software that allows students to understand chemistry more easily. Another important objective of the project is the use of algorithms for determining oxidation numbers and linear algebra in the process of establishing the final coefficients of a redox equation.

\section{RELATED WORKS}

Henry J. Brown provides a brief history of the evolution of games, from Plato to the present, in his book "Videogames and Education" [4]. Plato described the game as a form of prayer. Throughout history, games have been directly influenced by major events in history, being closely linked to people's lives and helping them to develop their thinking, imagination, or ability to adapt to different situations. The book also defines the concept of "edutainment", which refers 
to a combination of education and entertainment. The first company to use this term was Electronic Arts, when it launched the game Seven Cities of Gold in 1984.

Selvi and Çoșan [5] conducted a study to determine the impact of a video game on learning concepts related to the plant and animal kingdoms. The study showed that participants became more interested in the subject matter and the learning rate increased. Games are more motivating in terms of performing a learning task.

E. Klopfer [6] describes in the book "Augmented Learning: Research and Design of Mobile Educational Games" the importance of mobile games in the learning process. Mobile games allow connection to the real world through augmented reality and offer a new vision of how technology can be used to stimulate cognitive development. A constructive approach can be taken to create an interactive game where the user is rewarded for each correct answer. But the way this game is constructed may still not have the desired outcome. In some cases users become more focused on the game itself. So all the knowledge gained is learned in a systematic way without actually being understood. Therefore when designing a game, development should be done using several approaches so that the final product is more focused on learning, not entertainment. Creativity and inventiveness are key factors in achieving a satisfactory result in this respect. Games based on augmented reality allow the creation of a more interactive environment through which the user is motivated and the educational goal is achieved.

In the book "Oxidation Numbers and Oxidation States" [7], Chr. K. Jorgensen describes how oxidation numbers are formed in chemical processes. At the same time, the fundamental axioms for defining and determining these numbers are presented in this work. For each axiom, the author gives a concrete example in which it applies. A set of rules for the determination of oxidation numbers is also given, in which special cases in the process of determining these numbers are also treated.

In the paper entitled "From Chemistry to Linear Algebra: Balancing a Chemical Reaction Equation Using Algebraic Approach" [8], the author describes a method for equating chemical equations via linear algebra using the MATLAB development environment. Using a linear system of equations and applying Gaussian elimination, it can reduce the time to determine stoichiometric coefficients for those very complex chemical equations.

\section{MARKER BASED AR}

This type of augmented reality uses marker objects to make the camera recognise augmentable objects. The virtual world is anchored in the real world, so to trigger the recognition algorithm, the camera must be pointed at a marker picture. To achieve this, static images are used, so when scanning, virtual objects, text, animations or sound are superimposed over the real world.

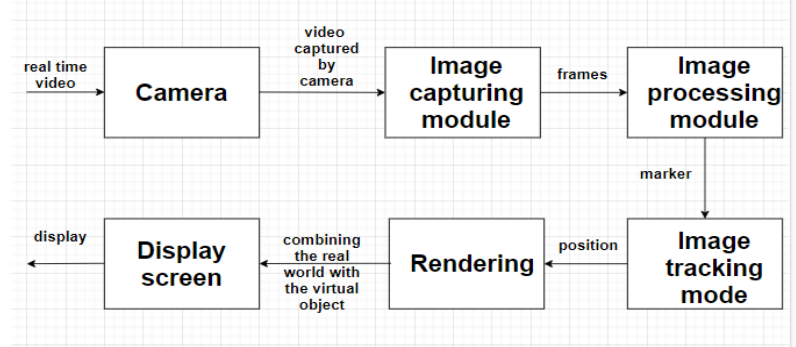

Figure 1 Processing steps for marker object detection [10]

Marker-based augmented reality focuses on multiple successive processing [9]. The video captured by the camera is processed and transformed into frames. In each frame a processing of the captured image is performed and the marker position is calculated. The position of virtual objects depends entirely on the triggering objects. When this position is calculated, the interpretation of the objects is performed. Finally the virtual objects are included in the real scene and are placed, according to the previously calculated position. The most popular applications using marker-based AR are QR code scanning applications.

\section{Vuforia}

Unity has a framework called Vuforia that allows detection of both 2D and 3D objects and uses both marker-based and markerless models. The most widely used model in Vuforia is the marker-based model, as it allows defining target images or objects in a database that are recognised by the camera. In addition to these, this SDK provides many other features, such as: tracking target objects, handling application fragmentation using fusion, Cloud-based target recognition service or virtual graphical user interface elements.

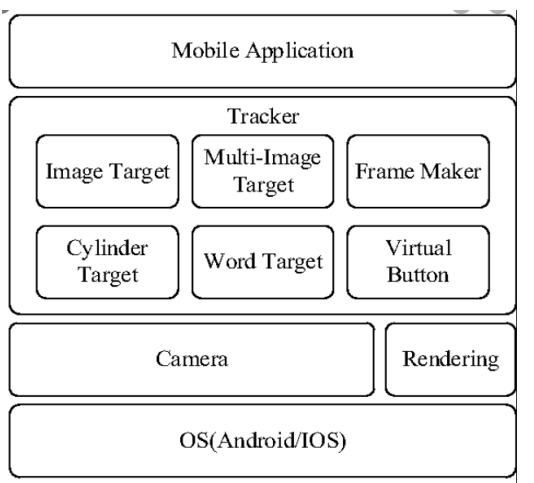

Figure 2 Architecture Vuforia [10]

Target objects can be defined in the database as image, cuboid, cylinder or $3 \mathrm{D}$ objects. For each object, the basic features that allow detection by the camera are searched for. The main feature used is the outline of the object, then there are colour and shadows. 


\section{Image Targets}

The app uses marker-based augmented reality, so it uses static images as target objects. These images are called Image Targets and must be declared in a database. Detection is done by means of contour and colour extraction, so the images declared in the database must contain as many elements as possible in order to extract features, but at the same time they must be as suggestive as possible for the user. Detection and tracking of target objects is done by comparing image features in the camera spectrum with image features in the database in order to superimpose the augmented object over the image. Image Target Manager allows these images to be defined in a database that can then be attached to the AR camera defined in the Unity scene.

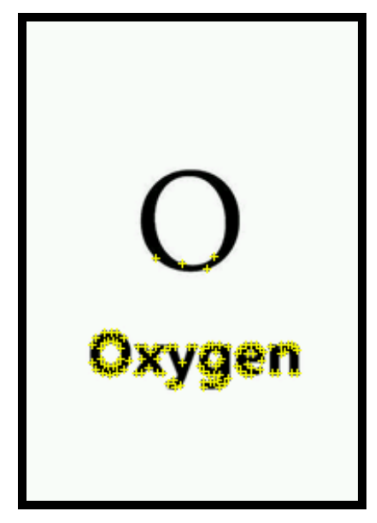

Figure 3 Sample features extracted from Image Target Manager

The camera works while the scene is on and captures image frames in real time. Each frame is processed and compared with the images defined in the database. Each chemical element is an Image Target object. When the camera detects a chemical element, the augmentable objects attached to it will be activated. These augmentable objects are intended to provide additional information about the detected chemical element (electronic configuration in the form of animated atoms, atomic mass, possible oxidation numbers, element type). At the same time, at the time of detection, another image will be superimposed on the static image, one as suggestive as possible to capture the user's attention.

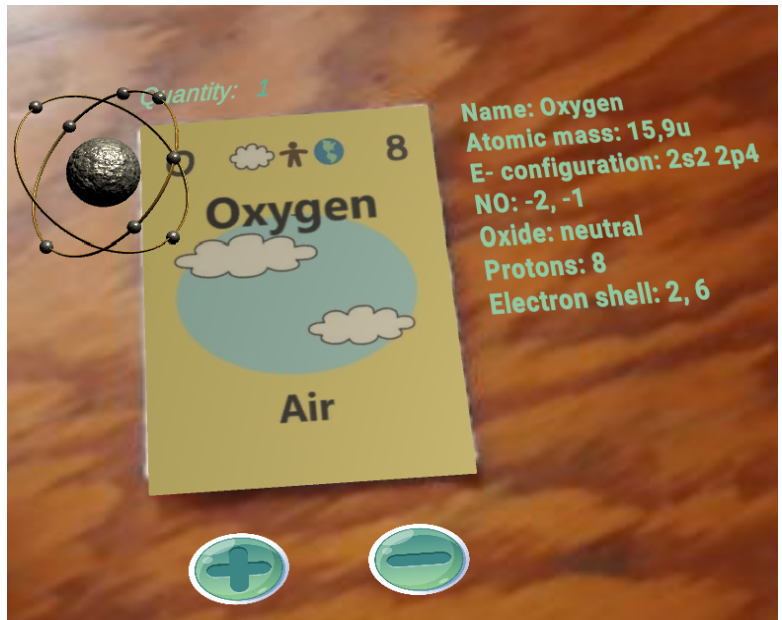

Figure 4 Generated augmentable objects.

\section{Composition of chemicals}

When two cards are close together, the resulting chemical compound is detected. To achieve this, Unity has two components called BoxCollider and RigidBody. Together they are able to detect collision between components. RigidBody allows detection of the type of collision, while BoxCollider detects the collision itself.

Chemical elements in a substance can have different amounts of molecules. To allow incrementing/decrementing the quantity, each static image contains two buttons for the specified functionalities. To uniquely identify each card detected in the scene, it will have a label attached with the name of the chemical element printed on the card.

Unity allows collision detection via the BoxCollider component which automatically calls the functions: OnTriggerEnter(Collider collider) and OnTriggerExit(Collider collider). So the moment two cards are close together, these functions are called, the chemical element labels and the number of related molecules are checked to activate a new augmentable element. This element contains an image conforming to the newly detected chemical and the other virtual objects attached to the chemical elements will be deactivated.

For example, if we want to detect the chemical compound $\mathrm{H}_{2} \mathrm{O}$ (water), we insert the hydrogen and oxygen cards. For the hydrogen card, we increase the quantity to two. The next step is to bring the two cards together, at which point the collision is detected and additional information about the chemical compound $\mathrm{H}_{2} \mathrm{O}$ is displayed. 


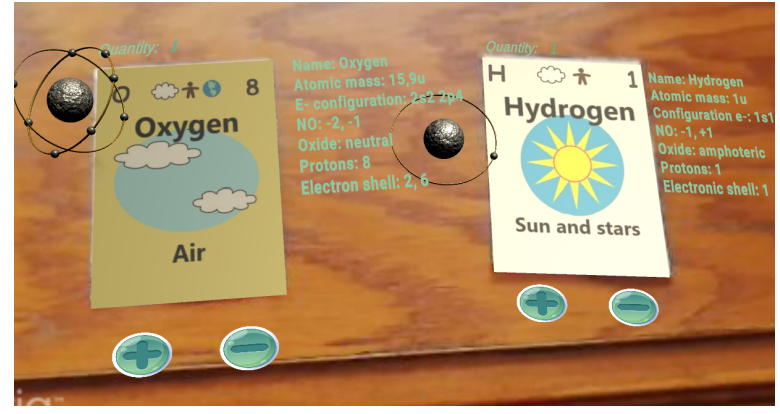

Figure 5 Hydrogen and oxygen detection

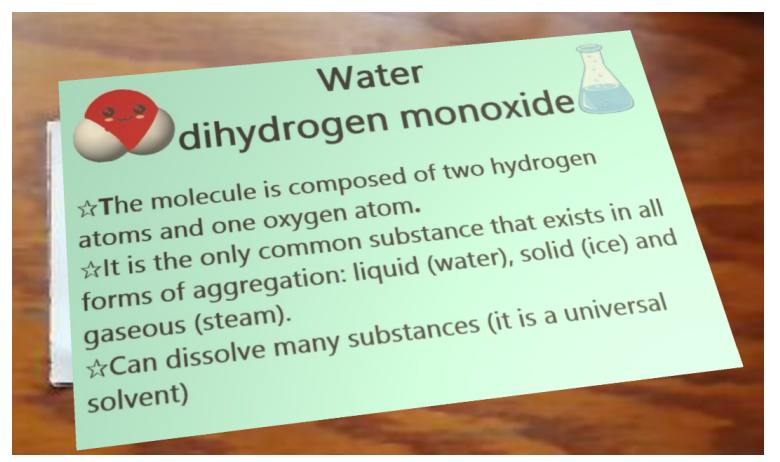

Figure 6 Detection of chemical compound water

\section{REDOX EQUATIONS}

Any chemical equation is made up of two components: reactants and products. The reactants are on the left-hand side of the equation, while the products are on the right-hand side. The two components are separated by the symbol ' $\rightarrow$ ' or ' $=$ '. Several elements or chemicals are involved in a chemical substance, each with a stoichiometric coefficient.

Redox equations are those chemical equations in which the oxidation numbers of atoms are changed. This type of equation consists of two processes: oxidation and reduction. If the chemical receives electrons, then the oxidation number decreases and reduction takes place. If the oxidation number increases, then the chemical gives up electrons and oxidation takes place.

\section{Establishment of oxidation numbers}

Oxidation numbers generally refer to the degree of oxidation of an atom [11] and play an important role in the formation of chemical reactions. They can be of three types: zero, negative or positive. Depending on the chemical compound they are part of, elementary chemical elements can have several oxidation numbers. A chemical compound is a chemical substance made up of several identical molecules of one atom. Each chemical involved in a chemical reaction can have only one oxidation number. To determine oxidation numbers, certain rules are defined that must be followed when assigning them [12]:

- The atoms of elementary molecular substances have an oxidation number equal to 0 (e.g. $\mathrm{I}_{2}, \mathrm{Cl}$ ).

- In ionic compounds, the elements have an oxidation number equal to the number of electrons received or given up in the ion formation process (e.g. $\mathrm{NaCl}$, $\mathrm{Na}$ has $\mathrm{O} . \mathrm{N} .=+1$ and $\mathrm{Cl}$ has $\mathrm{O} . \mathrm{N} .=-1)$.

- Hydrogen has an oxidation number equal to +1 in covalent compounds (e.g. $\mathrm{HCl}, \mathrm{H}_{2} \mathrm{O}$ ).

- Hydrogen has an oxidation number equal to -1 in ionic hydride compounds. Ionic hydrides are composed of alkali metals of the first or second main group. (e.g. $\mathrm{NaH}, \mathrm{CaH}_{2}$ ).

- Oxygen has oxidation number -2 , except for peroxides, where it has -1 (example of exceptions: $\mathrm{H}_{2} \mathrm{O}_{2}$ ).

- Monoatomic ions have an oxidation number equal to the charge of the ion.

- Polyatomic ions have the sum of their oxidation numbers equal to the charge of the ion. (e.g. $\mathrm{SO}_{4}$ has always $\mathrm{O} . \mathrm{N} .=-2$ ).

- Elements in the first main group have an oxidation number equal to +1 (e.g. Li, Na, K, Rb, Cs, Fr).

- Elements in the second main group have an oxidation number equal to +2 (e.g. $\mathrm{Mg}, \mathrm{Ca}, \mathrm{Sr}, \mathrm{Ba}$ ).

- Elements in the seventh main group have an oxidation number equal to -1 (e.g. $\mathrm{Cl}, \mathrm{Br}, \mathrm{I}, \mathrm{F}$ ).

- Atoms of elements in neutral molecules have sum of oxidation numbers equal to 0 . (e.g. $\mathrm{H}_{2} \mathrm{SO}_{4}$, we know that $\mathrm{SO}_{4}$ has $\mathrm{O}$. $\mathrm{N} .=-2$, so we have the equation $0=\mathrm{O}$. N.so4 $+2 *$ O.N.H. From thi we get $\mathrm{O}$. $\mathrm{N} \cdot \mathrm{H}=+1)$

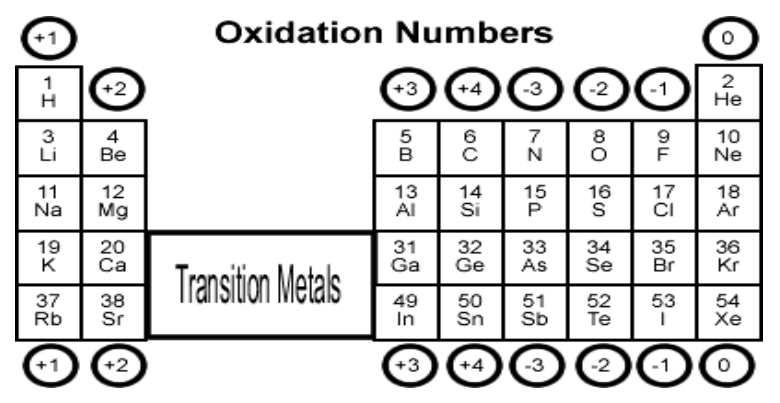

Figure 7 Possible oxidation numbers for certain chemical elements [13] 
These rules are important and must be applied in the order in which they are defined. If this order is not followed, the oxidation numbers cannot be determined correctly.

\section{Methods for equating redox equations}

Chemical redox equations can be equated by two methods: using oxidation numbers or using the ionic equation. Both methods use oxidation numbers, but the ionic equation involves determining the acidic or basic medium in which electronic transfer takes place. The ionic equation method is more complex and harder to understand, so the approach chosen for this project is the oxidation number equation method.

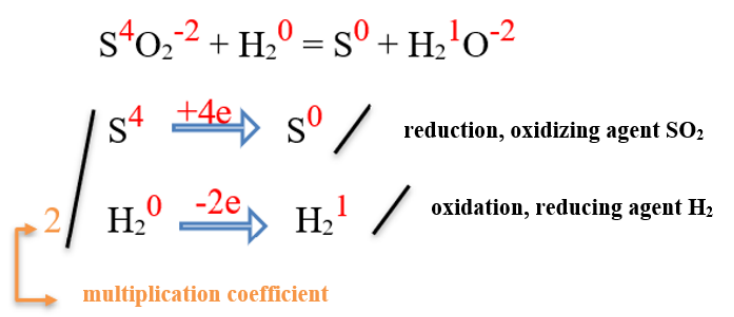

Figure 8 Redox equation using oxidation numbers

For this method the equation is divided into two halfreactions: products and reactants. For each element in the half-reaction the oxidation number is determined. In the next step, the oxidising agent (the substance that receives electrons) and the reducing agent (the substance that gives up electrons) are identified. In any chemical reaction, the number of electrons received must equal the number of electrons given up, so the lowest common multiple of electrons accepted and electrons given up is sought. Each half-reaction is multiplied by the previously calculated number. The multiplication coefficients in the redox halfreactions are at the same time the stoichiometric coefficients for the chemical reaction process. The final step of the equation is to determine the coefficients for the other chemical species involved in the chemical process.

Depending on which chemicals accept or give up electrons, chemical reactions can be [14]:

- Intramolecular: electron exchange between different atoms belonging to the same molecule

- Dismutation: the exchange of electrons takes place between identical atoms belonging to the same molecule.

- Intermolecular: electron exchange between different atoms belonging to different molecules

The case of dismutation reactions is a special case and must be treated separately. Since the transfer is between atoms in the same molecule, after calculating the lowest multiple, the coefficients of the redox half-reactions are added together to determine the multiplication coefficient. This sum represents the stoichiometric coefficient in the main chemical reaction.

\section{Establishment of final stoichiometric coefficients}

The final step in redox equalisation involves determining the stoichiometric coefficients for the other chemical species. Chemical reactions can be equated by several methods, including the inspection method or using linear algebra.

The inspection method is one of the most widely used methods. This method is based on 'guessing' the stoichiometric coefficients for each chemical element involved in the chemical reaction. Essentially successive guesses are made about the stoichiometric coefficients for a particular chemical element until a correct final result is reached. There are certain rules that facilitate the determination of these coefficients.

Equating a chemical equation using linear algebra involves constructing a system of $\mathrm{n}$ unknowns and $\mathrm{m}$ equations. To construct the matrix of the system, the elementary chemical elements participating in the equation must be determined. Their number determines the number of rows in the matrix. To determine the number of columns, the number of chemicals involved in the chemical reaction is calculated.

After the matrix dimensions have been determined, the matrix is filled in. For each chemical element $x$, we determine the number of atoms that are in chemical $y$. This number represents the value in column $\mathrm{x}$ and row $\mathrm{y}$. After determining the matrix elements, we apply Gaussian elimination to simplify the equation system and obtain an echelon matrix. A matrix is echelon if [15]:

- All rows below the main diagonal are zero

- The leading coefficient or pivot of a non-zero row is to the right of a leading coefficient of the row above it.

Gaussian elimination involves applying finite sequences of elementary operations on the matrix (additions and subtractions). Application of this method results in a system of equations in which the variables corresponding to the pivot are constants or linear functions of the other variables, if they exist. For linear systems generated from chemical equations, variables in the form of fractions are obtained. But the stoichiometric coefficients are integers. To eliminate fractions, we determine the lowest common multiple of all the denominators of the variables that are fractions. If the other variables corresponding to the pivot depend on a constant, we multiply this constant by the value of the lowest multiple calculated. The same procedure is performed if the other variables depend on another variable. If they depend on more than one variable, we make a linear system of the free variables and calculate the determinant to find their values.

For the equation $\mathrm{SO}_{2}+\mathrm{H}_{2}=\mathrm{S}+\mathrm{H}_{2} \mathrm{O}$ the following linear system is obtained: a $\mathrm{SO}_{2}+\mathrm{b} \mathrm{H}_{2}=\mathrm{c} \mathrm{S}+\mathrm{d} \mathrm{H}_{2} \mathrm{O}$. 
The steps for obtaining stoichiometric coefficients are described in the table below:

\begin{tabular}{|l|l|l|}
\hline Equation system & Operations & Related matrix \\
\hline $\begin{array}{l}a-c=0 \\
2 a-d=0 \\
2 b-2 d=0\end{array}$ & {$\left[\begin{array}{llll}1 & 0 & -1 & 0 \\
2 & 0 & 0 & -1 \\
0 & 2 & 0 & -2\end{array}\right]$} \\
\hline $\begin{array}{l}a-c=0 \\
2 c-d=0 \\
2 b-2 d=0\end{array}$ & L2-2L1 $\rightarrow$ L2 & {$\left[\begin{array}{rrrr}1 & 0 & -1 & 0 \\
0 & 0 & 2 & -1 \\
0 & 2 & 0 & -2\end{array}\right]$} \\
\hline $\begin{array}{l}a-c=0 \\
2 b-2 d=0 \\
2 c-d=0\end{array}$ & L2 $\rightarrow$ L3 & {$\left[\begin{array}{rrrr}1 & 0 & -1 & 0 \\
0 & 2 & 0 & -2 \\
0 & 0 & 2 & -1\end{array}\right]$} \\
\hline $\begin{array}{l}a-c=0 \\
b-d=0 \\
c-1 / 2 d=0\end{array}$ & $1 / 2 L 2 \rightarrow$ L2 & {$\left[\begin{array}{rrrr}1 & 0 & -1 & 0 \\
0 & 1 & 0 & -1 \\
0 & 0 & 1 & -\frac{1}{2}\end{array}\right]$} \\
\hline $\begin{array}{l}a-1 / 2 d=0 \\
b-d=0 \\
c-1 / 2 d=0\end{array}$ & L3+L1 $\rightarrow$ L1 & {$\left[\begin{array}{rrrr}1 & 0 & 0 & -\frac{1}{2} \\
0 & 1 & 0 & -\frac{1}{1} \\
0 & 0 & 1 & -\frac{1}{2}\end{array}\right]$} \\
\hline
\end{tabular}

Figure 9 Steps in Gaussian elimination

This gives the equations:

$$
\left\{\begin{array}{c}
a=\frac{1}{2} d \\
b=d \\
c=\frac{1}{2} d
\end{array}\right.
$$

The least common multiple of all the denominators of the variables that are fractions is 2 . So the variable $d=2$ is chosen. This gives the system:

$\left\{\begin{array}{l}a=1 \\ b=2 \\ c=1 \\ d=2\end{array}\right.$

The final equation is: $\mathrm{SO}_{2}+2 \mathrm{H}_{2}=\mathrm{S}+2 \mathrm{H}_{2} \mathrm{O}$.

Although the inspection method is one of the most widely used methods, it is not the most effective. This method works very well and is easy to apply to very simple chemical reactions. If the chemical equation becomes more complex, this method is impossible, resource-intensive and timeconsuming. If assumptions are not made correctly, wrong answers can be generated that cannot be detected early. The application wants to be able to respond as quickly as possible to the user's requests to match redox reactions, regardless of their complexity. That is why the linear algebra method is used. This method is much faster, does not depend on the complexity of the chemical equation and generates correct answers in all cases. Computationally, this method does not require a lot of resources and processing time.

\section{IMPLEMENTATION DETAILS}

The app was developed using Unity, which allows game development for many platforms: Windows, Linux, Android, iOS, etc. The code part was written in $\mathrm{C \#}$.

The application consists of four modules, each with functional components and corresponding objects. The most important modules of the application are learning module and testing module. In the learning module the user can use augmented reality technology to learn additional information about chemical elements and substances with the help of cards. Also in this module is the functionality for equating chemical equations, along with the display of intermediate steps for determining oxidation numbers. The test module aims to check the knowledge the user has gained from using the learning module. This module consists of nine questions of varying difficulty: easy, medium and hard. It works in such a way that when the user answers a question correctly, his/her mark is increased without being penalised if he/she gets the answer wrong. The two modules use the AR camera to detect the cards. In the test module the answers for the question are only displayed when the camera detects the card corresponding to the chemical in the question.

In order to apply the methods for determining oxidation numbers and linear system formation to equate the redox equation, the chemical elements must be extracted from equation, along with the quantities of atoms. For this purpose, the Atom class was created in which the information about each chemical substance (name, number of molecules, oxidation number) is kept. Regular expressions are used for this to extract chemicals into a list of Atoms. Substances are treated differently, depending on whether they are simple or compound. For simple chemicals, the number of atoms is extracted directly from the chemical symbol. Compounds must be divided into simple substances using regular expressions. Each rule for determining the oxidation number was implemented and applied to each element in the list of Atoms. 


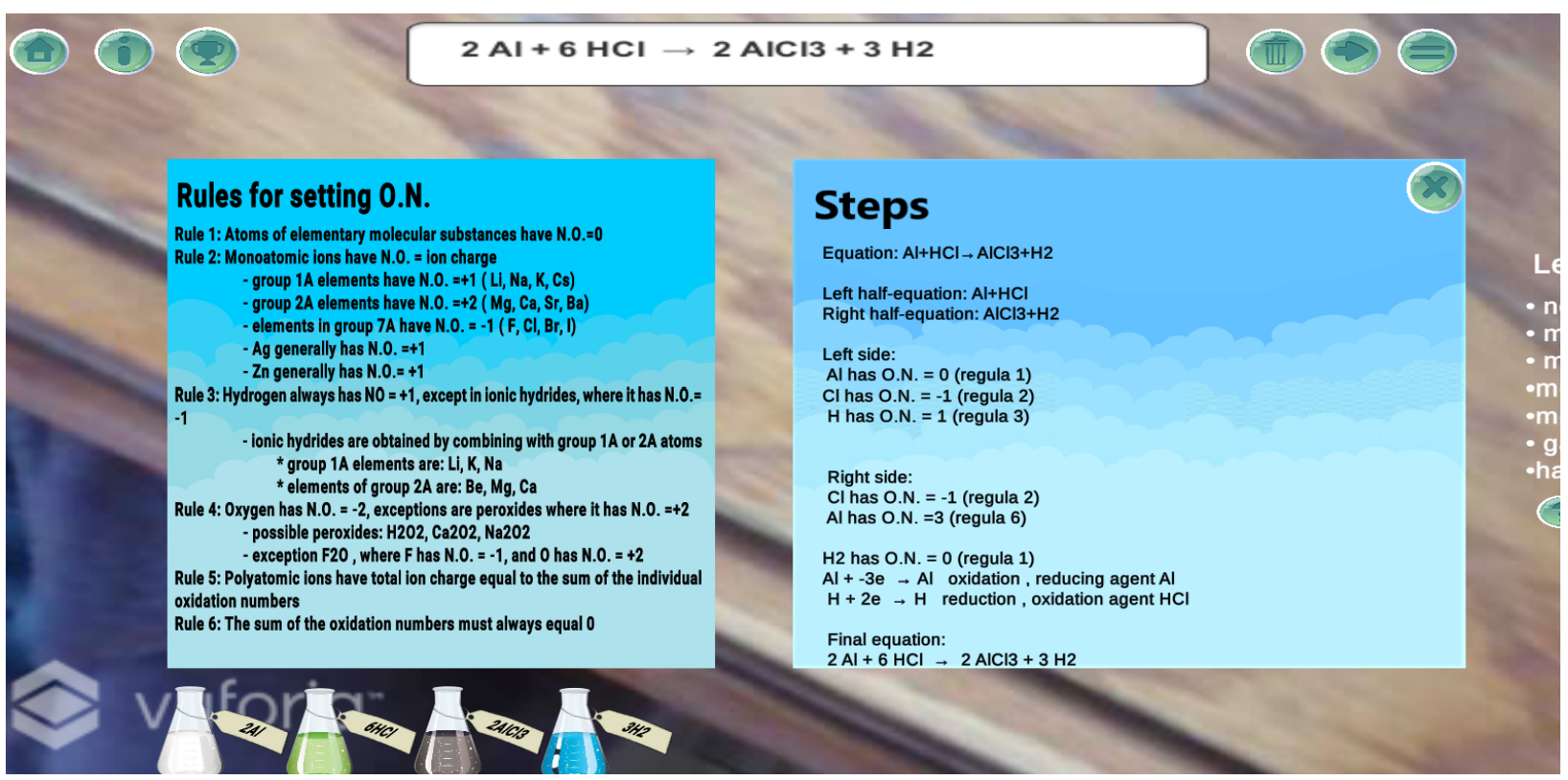

Figure 10 Equating chemical reactions and setting oxidation numbers

\section{System requirements}

The app runs on Android and uses augmented reality. The following were used to implement the project:

- Visual Studio IDE for writing C\# scripts

- Unity 2019.3.13f1

- Vuforia Package

- $\quad$ Android SDK

- Version of Unity Hub enabling Android support.

- A device with a working camera connected to the app

\section{TESTING}

\section{Functional testing}

Functional testing was used in the project to test how the application responds when the user performs an action that changes the state of the system. Testing was performed iteratively with the aim of troubleshooting errors in the development process and improving the system architecture. User can change the state of system by interacting with UI elements, introducing a card in the camera's field of view or composing the chemical elements through multiple cards.

Card detection is directly dependent on Vuforia Target Manager and how it can find features for a particular image. Several sets of postcards were chosen and tests were run until the camera was able to recognise all of them in a short time. Tests were also carried out for the collision detection part and the generation of questions and answers in the test module.
At the same time tests were performed to verify the functionality of setting oxidation numbers and equating redox equations, regardless of their complexity. Following the tests performed, the application was able to meet all requirements.

\section{User testing}

One of the most important tests carried out was user testing. This aimed to test the effectiveness of the application in understanding and equating redox reactions. The app was tested with the help of a chemistry teacher and 9th grade students. The interaction between the app and the user and the opinion that the user forms as a result of its use was followed. Users were provided with the set of cards.

After testing the app, users were asked to fill in a form to give their opinion on PlayChemy. Both the students and the chemistry teacher were delighted with the idea of the app. They were asked if they found the app easy to use, quite suggestive in learning oxidation numbers and equating redox reactions. In their opinion, the students would use the app as a practical support for the lessons in the redox chapter and the teacher would use the app as a teaching aid for these lessons. As a general conclusion of both students and teacher, the fact that the app is able to specify exactly which rule was applied when determining the oxidation numbers is a great advantage in the process of understanding these numbers. The method of equating redox equations preferred by users is the oxidation numbers. This is because this method is much easier to understand and use, unlike the ionic equation method which is much more complex. 


\section{CONCLUSIONS}

Video games and simulators have been increasingly used in education. Especially in an online classroom support scenario, video games have proven to be useful in learning and understanding theoretical concepts. Games based on augmented reality technology have been created especially for pupils or students who need help in understanding terms in the space of engineering problems, mathematics or geometry. With accurate reconstructions of concepts in certain fields, AR games have proven to be very useful in the process of assimilating and understanding these concepts.

PlayChemy uses AR technology to create an environment in which the student can deepen their knowledge of certain elementary chemistry concepts or redox equations. The app is intended for both students and teachers. Teachers can use the app to make their chemistry lessons more interactive and engaging for students.

In terms of elementary video games, augmented reality technology-based games have recently been introduced in the educational field. PlayChemy wants to come up with something new in this field, creating an opportunity especially for students in the field of chemistry. Using augmented reality, the app opens up new opportunities for experimenting and understanding chemistry concepts using cards inscribed with chemical elements. Compared to other apps such as Arloon Chemistry, PlayChemy allows to detect chemicals using multiple cards. Redox Reaction Chemistry is a system that allows redox equalization without displaying the intermediate steps and does not use augmented reality, unlike PlayChemy.

Further developments of the application could be: detection of the composition of chemical substances between several elementary substances, extension of the learning and testing modules with other essential chapters of the chemistry curriculum.

\section{REFERENCES}

1. M. De Aguilera and A. Mendiz Noguero, „Video games and education: (Education in the face of a "parallel school")," Computers in Entertainment, 2003.

2. J. Clement, "Most popular devices used for video games worldwide as of January 2021," Statista, March 2021. [Online]. Available:

https://www.statista.com/statistics/1132960/populardevices-video-games/. [Accessed February 2021].

3. Wikipedia, "Augmented reality," Wikipedia, May 2020. [Online]. Available:
https://en.wikipedia.org/wiki/Augmented_reality. [Accessed February 2021].

4. H. J. Brown, Videogames and Education, M.E. Sharpe, 2008

5. M. Selvi and A. Çoşan, ,,The Effect of Using Educational Games in Teaching Kingdoms of Living Things," Universal Journal of Educational Research, vol. 6, 2018.

6. E. Klopfer, Augmented Learning: Research and Design of Mobile Educational Games, MIT Press, 2008

7. C. K. Jorgensen, Oxidation Numbers and Oxidation States, Springer Science \& Business Media, 2012, 6-12

8. Zabadi, ,FROM CHEMISTRY TO LINEAR ALGEBRA: BALANCING A CHEMICAL REACTION EQUATION USING ALGEBRAIC APPROACH," International journal of advanced biotechology an research (UBR), vol. 8, 2017.

9. X. Cheng and Z. Lifeng, „Implementation of mobile augmented reality based on Vuforia and Rawajali," IEEE 5th International Conference on Software, pp. 912-915, 2014.

10. J. Linowes and K. Babilinski, Augmented Reality for Developers, Pakt, 2017

11. Wikipedia, „Oxidation state,” Wikipedia, 2008. [Interactiv]. Available: https://en.wikipedia.org/wiki/Oxidation_state. [Accessed March 2021].

12. R. Le Roy, „RULES FOR ASSIGNING OXIDATION STATES," Scienide2, [Interactiv]. Available: http://scienide2.uwaterloo.ca/ rleroy/c120/oxid_state_ RULES.pdf [Accessed March 2021].

13. Socratic, ,What are the possible oxidation numbers of carbon?," Socratic, 2015. [Interactiv]. Available: https://socratic.org/questions/56204bef1 1ef6b2fb682c4 f1. [Accessed March 2021].

14. Britannica, ,Intermolecular reaction,” Britannica, 2017. [Interactiv]. Available:

https://www.britannica.com/science/intermolecularreaction. [Accessed March 2021].

15. Wikipedia, „Row echelon form,” Wikipedia, 2008. [Interactiv]. Available: https://en.wikipedia.org/wiki/Row_echelon_form. [Accessed March 2021] 